\title{
The relationship between glycemic control and BNP levels in diabetic patients
}

\author{
Kursat Dal ${ }^{1}$, Naim Ata ${ }^{1}$, Bunyamin Yavuz ${ }^{2}$, Omer Sen ${ }^{2}$, Onur Sinan Deveci ${ }^{2}$, \\ Zekeriya Aksoz ${ }^{1}$, Aslihan Mete Yildirim, Bulent Uygungelen ${ }^{3}$, \\ Kadir Okhan Akin ${ }^{4}$, Esin Beyan $^{1}$, Derun Taner Ertugrul ${ }^{1}$ \\ ${ }^{1}$ Department of Internal Medicine, Kecioren Teaching and Research Hospital, Ankara, Turkey \\ ${ }^{2}$ Department of Cardiology, Kecioren Teaching and Research Hospital, Ankara, Turkey \\ ${ }^{3}$ Department of Internal Medicine, Andirin State Hospital, Kahramanmaras, Turkey \\ ${ }^{4}$ Department of Biochemistry, Kecioren Teaching and Research Hospital, Ankara, Turkey
}

\begin{abstract}
Background: Glycemic control affects cardiovascular risk factors positively. The purpose of this study was to assess B-type natriuretic peptide (BNP) levels in patients with poorly controlled diabetes before and after glycemic regulation was achieved.

Methods: The study was performed in a prospective design. The study population consisted of 79 consecutive diabetic patients with poor glycemic control. All subjects underwent transthoracic echocardiography. Levels of fasting plasma glucose, glycosylated hemoglobin (HbA1c), lipid parameters, and BNP were measured before the onset of the treatment and after glycemic regulation was achieved.

Results: A significant decrease in BNP (95.0 [4.0-1807] ng/L vs. 52.0 [2.1-987.0] ng/L, $p<$ 0.001) levels were observed, after improving glycemic control. The decrease in BNP levels was positively correlated with the decrease in HbA1c $(r=0.345, p=0.003)$ and fasting plasma glucose $(r=0.366, p=0.002)$. There was no correlation between the decrease in BNP levels and lipid parameters $(p=N S)$.

Conclusions: We conclude that poor glycemic control may cause high levels of BNP which may lead to overdiagnosis of congestive heart failure. We suggest that HbA1c and fasting plasma glucose should be checked in patients with high levels of BNP. (Cardiol J 2014; 21, 3: 252-256)
\end{abstract}

Key words: glycemic control, diabetes, BNP, echocardiography, heart failure

\section{Introduction}

Glycemic optimization is a crucial part of management in type 2 diabetes. It can be achieved by diet, weight loss, oral anti-diabetics and insulin therapy. Improving glycemic control positively affects cardiovascular risk factors such as plasma lipids, thrombotic markers, and blood pressure [1-4].
B-type natriuretic peptides (BNP) are produced in ventricular cardiomyocytes, and secreted in response to volume expansion or pressure overload. The BNP test is widely used to diagnose preclinical heart disease or to confirm the cardiac etiology in symptomatic patients. BNP can be affected by several extra-cardiac conditions such as rheumatic diseases, pulmonary diseases [5, 6].

Address for correspondence: Bunyamin Yavuz, MD, Assoc. Prof., Department of Cardiology, Kecioren Teaching and Research Hospital, Kecioren, Ankara, Turkey, e-mail: byavuzmd@gmail.com 
Although there are several studies evaluating the effect of glycemic control on some biochemical markers such as lipids in diabetic patients, there is a limited number of controversial studies assessing the effect of the glycemic control on the BNP levels. The aim of this study is to evaluate the relationship between the glycemic control and the BNP levels.

\section{Methods}

The study was performed in a prospective design. The study population consisted of 79 consecutive diabetic patients with poor glycemic control defined as glycosylated hemoglobin (HbA1c) $\geq 8.5 \%(69 \mathrm{mmol} / \mathrm{mol})$.

Exclusion criteria were the presence of coronary artery disease, overt heart failure (using Framingham criteria), left ventricular systolic dysfunction (ejection fraction $<40 \%$ ), pregnancy or lactation, hepatic or renal dysfunction, significant neurological or psychological diseases, inflammatory bowel diseases, and systemic autoimmune diseases such as systemic lupus erythematosus, rheumatoid arthritis, and scleroderma. Changes in antihypertensive and anti-lipidemic drugs were exclusion criteria for the study. The study protocol is in accordance with the Declaration of Helsinki. The Local Ethics Committee approved the study protocol. All patients gave their informed consents.

All subjects underwent an initial screening assessment that included a medical history and physical examination. All subjects were hospitalized for the glycemic regulation. All subjects were administered the same type of analogue insulin treatment $\left(\right.$ Novomix $^{\circledR}$, Novonordisk ${ }^{\circledR}$ ) for glycemic regulation. All subjects underwent transthoracic echocardiography before and after insulin treatment. Fasting plasma glucose, HbA1c, lipid parameters, BNP, renal and liver function tests, electrolytes, were obtained at baseline and after 2 weeks of insulin treatment.

Fasting blood samples were obtained by the venipuncture of large antecubital veins of the studied patients without stasis, after a 12 -h fast. The samples were then centrifuged immediately; the plasma was separated and stored at $-80^{\circ} \mathrm{C}$. In order to avoid variation, all samples were studied on the same day and with the same kit. Total cholesterol (Lot No: B540, Konelab) and triglyceride (Lot No: C186, Konelab) were measured with enzymatic colorimetric tests. Low-density lipoprotein-cholesterol (LDL-C) (Lot No: C435, Konelab) and high-density lipoprotein-cholesterol (HDL-C) (Lot No: C136, Konelab) were measured with the homogeneous enzymatic colorimetric test. $\mathrm{HbA1c}$ measurements were performed by ion-exchange high-performance liquid chromatography with Tosoh G7 (Tosoh, Grove City, OH).

The concentrations of BNP were determined with a 2 -site sandwich chemiluminescence immunoassay (Lot No.: 22161145, reference No.: 02816634) on the ADVIA $^{\circledR}$ Centaur $^{\circledR}$ platform (Siemens Healthcare Diagnostic, IL, USA). The ADVIA Centaur BNP assay is a fully automated 2 -site sandwich immunoassay using direct chemiluminescent technology, which applies constant amounts of 2 monoclonal antibodies. The first antibody, in the Lite reagent, is acridinium ester labeled monoclonal mouse anti-human BNP F(ab') $\times$ fragment specific to the ring structure of BNP. The second antibody, in the solid phase, is a biotinylated monoclonal mouse anti-human antibody specific to the C-terminal portion of BNP, which is coupled to streptavidin magnetic particles. The limit of detection for this assay was $0.5 \mathrm{ng} / \mathrm{L}$ and showed that the within-run and total imprecision coefficient of variation was $3.5 \%$. Values less than $0.5 \mathrm{ng} / \mathrm{L}$ were regarded as non-detectable.

Transthoracic echocardiography: Transthoracic echocardiography examination was performed to all subjects by using a System Three (GE Vingmed Ultrasound, Horten, Norway) cardiac ultrasound scanner and 2.5-3.5 MHz transducers.

Echocardiography of the left ventricle: The following parameters were measured from cross-sectional echocardiographic images of the left ventricle: 1) end-diastolic and end-systolic diameter [cm]; 2) fractional shortening (FS \%); 3) ejection fraction $(\mathrm{EF} \%)$.

Doppler echocardiography: Flow velocity indexes were obtained using pulsed and continuous wave Doppler from apical projections, and measurements were made utilizing the software of the ultrasound equipment. Mitral diastolic flow was obtained after the pulsed Doppler sample volume was positioned perpendicularly to the tips of the mitral valve leaflets. The Doppler cursor was then moved towards the left ventricular outflow position, and the sample volume was placed approximately $1 \mathrm{~cm}$ proximally to the aortic valve so that it would come in contact with the anterior mitral valve leaflet. Isovolumic relaxation time [ms] was measured as the interval between the end of the aortic click artifact and the onset of mitral inflow waveform.

The following indexes were measured from the mitral and tricuspid valve diastolic wave form: peak early (E) and atrial (A) flow velocities [m/s], $\mathrm{E} / \mathrm{A}$ ratio of the left ventricular diastolic filling. Heart rate [bpm] was measured from simultaneous electrocardiogram recordings. 
Pulsed Doppler tissue echocardiography: The myocardial velocities of the left ventricle were measured sampling the mitral annulus excursion at lateral sites in the 4-chamber view. Care was taken to keep the ultrasound beam perpendicular to the plane of the annulus in order to minimize the angle between the beam and the direction of annular motion. The width of the sample volume was $3-5 \mathrm{~mm}$. Measurements were focused on the systolic myocardial wave ( $\left.\mathrm{S}^{\prime}\right)$, the early-diastolic (E') and end-diastolic myocardial (A') waves. Usually, several cardiac cycles were acquired and the best 2 consecutive ones were analyzed and averaged.

\section{Statistical methods}

Distribution of the continuous variables was determined by the Kolmogorov-Smirnov test. Continuous variables with normal distribution were expressed as mean $\pm \mathrm{SD}$, variables with skew distribution are expressed as median (minimum-maximum), and categorical variables are expressed as percentages. The paired sample t-test was used for normally distributed variables and the Wilcoxon rank-sum test for skew distributed variables. Pearson and Spearman analysis was used to identify correlations between study parameters. For all statistics, a 2 -sided p value $<0.05$ was considered statistically significant. All analyses were performed with SPSS 10.0 for Windows.

\section{Results}

A total of 79 diabetic patients with poor glycemic control were examined. Demographic data, clinical characteristics, and medications are shown
Table 1. Demographic characteristic and medications of subjects.

\begin{tabular}{lc}
\hline Characteristic & Patients $(\mathbf{n}=\mathbf{7 9})$ \\
\hline Age [years] & $60.3 \pm 11.3$ \\
Female/Male & $53 / 26(67 / 33 \%)$ \\
Hypertension & $54(68 \%)$ \\
Medications and treatments: & \\
$\quad$ Sulfonylurea & $18(23 \%)$ \\
Metformin & $52(66 \%)$ \\
Thiazolidinediones & $21(27 \%)$ \\
Dipeptidyl peptidase-4 & $20(25 \%)$ \\
inhibitors & \\
\hline
\end{tabular}

in Table 1 . The mean age was $60.3 \pm 11.3$ years. The majority of patients were female $(53 ; 67 \%)$. Mean duration of diabetes was $7.5 \pm 3.5$ years. Systolic blood pressure and diastolic blood pressure were significantly decreased after improving glycemic control. There was no significant change in heart rate.

All subjects had normal ejection fraction. The transthoracic echocardiographic parameters of subjects are presented in Table 2. No significant difference was found in conventional Doppler and pulsed wave tissue Doppler parameters before and after glycemic control. There was no association between the measured echocardiographic indexes and BNP levels.

Biochemical parameters before and after glycemic control are presented in Table 3. Fasting plasma glucose levels $(306.3 \pm 119.4 \mathrm{mg} / \mathrm{dL}$ vs. $122.5 \pm 35.0 \mathrm{mg} / \mathrm{dL}, \mathrm{p}<0.001)$, HbA1c levels

Table 2. Conventional and tissue Doppler parameters of subjects before and after glycemic control.

\begin{tabular}{|c|c|c|c|}
\hline & Baseline & 2 weeks of glycemic control & $\mathbf{P}$ \\
\hline Left ventricular end-diastolic diameter [cm] & $4.6 \pm 0.7$ & $4.5 \pm 0.6$ & NS \\
\hline Left ventricular end-systolic diameter [cm] & $3.1 \pm 0.3$ & $3.0 \pm 0.2$ & NS \\
\hline Left ventricular ejection fraction [\%] & $66.3 \pm 4.7$ & $64.3 \pm 5.1$ & NS \\
\hline Left ventricular septal wall thickness [cm] & $1.1 \pm 0.2$ & $1.1 \pm 0.2$ & NS \\
\hline Left ventricular posterior wall thickness $[\mathrm{cm}]$ & $1.0 \pm 0.1$ & $1.0 \pm 0.1$ & NS \\
\hline E peak $[\mathrm{m} / \mathrm{s}]$ & $0.72 \pm 0.11$ & $0.76 \pm 0.13$ & NS \\
\hline A peak $[\mathrm{m} / \mathrm{s}]$ & $0.37 \pm 0.18$ & $0.41 \pm 0.23$ & NS \\
\hline E deceleration time [ms] & $209.1 \pm 31.4$ & $205.4 \pm 28.1$ & NS \\
\hline Isovolumic relaxation time [ms] & $91.3 \pm 12.8$ & $89.9 \pm 11.4$ & NS \\
\hline $\begin{array}{l}\text { Mitral early diastolic annular velocity } \\
\text { of lateral wall }\left(E^{\prime}\right)[\mathrm{cm} / \mathrm{s}]\end{array}$ & $12.6 \pm 2.5$ & $13.1 \pm 3.2$ & NS \\
\hline $\begin{array}{l}\text { Mitral late diastolic annular velocity } \\
\text { of lateral wall }\left(\mathrm{A}^{\prime}\right)[\mathrm{cm} / \mathrm{s}]\end{array}$ & $9.1 \pm 2.1$ & $8.4 \pm 2.7$ & NS \\
\hline $\begin{array}{l}\text { Mitral peak systolic annular velocity } \\
\text { of lateral wall }\left(\mathrm{S}^{\prime}\right)[\mathrm{cm} / \mathrm{s}]\end{array}$ & $12.5 \pm 2.7$ & $11.7 \pm 2.6$ & NS \\
\hline
\end{tabular}


Table 3. Biochemical and clinical parameters before and after glycemic control.

\begin{tabular}{lccc}
\hline & Baseline & 2 weeks of glycemic control & P \\
\hline Systolic blood pressure [mm Hg] & $128.2 \pm 9.8$ & $122.0 \pm 2.5$ & $<0.001$ \\
Diastolic blood pressure [mm Hg] & $82.0 \pm 7.8$ & $77.0 \pm 2.5$ & $<0.001$ \\
Heart rate [bpm] & $72.6 \pm 10.4$ & $72.8 \pm 11.2$ & $\mathrm{NS}$ \\
Fasting plasma glucose [mg/dL] & $306.3 \pm 119.4$ & $122.5 \pm 35.0$ & $<0.001$ \\
Triglyceride [mg/dL] & $199.5 \pm 118.4$ & $168.7 \pm 92.2$ & $<0.001$ \\
Total cholesterol [mg/dL] & $227.2 \pm 47.3$ & $207.2 \pm 45.2$ & $<0.001$ \\
Low density lipoprotein-cholesterol [mg/dL] & $151.3 \pm 34.3$ & $139.8 \pm 27.8$ & $<0.001$ \\
High density lipoprotein-cholesterol [mg/dL] & $42.7 \pm 13.7$ & $42.2 \pm 14.8$ & $\mathrm{NS}$ \\
Gama glutamyl transferase [U/L] & $32.3 \pm 22.0$ & $28.4 \pm 13.0$ & $\mathrm{NS}$ \\
Alanine aminotranferease [U/L] & $21.7 \pm 8.5$ & $22.6 \pm 9.7$ & $\mathrm{NS}$ \\
Aspartate aminotransferase [U/L] & $22.2 \pm 7.6$ & $21.6 \pm 9.9$ & $\mathrm{NS}$ \\
Creatinine [mg/dL] & $0.97 \pm 0.42$ & $0.96 \pm 0.48$ & $\mathrm{NS}$ \\
Glycosylated hemoglobin [\%] & $12.1 \pm 2.7(109 \pm 6)$ & $11.0 \pm 2.5(86 \pm 4)$ & $<0.001$ \\
\hline
\end{tabular}

$(12.1 \pm 2.7 \%[109 \pm 6 \mathrm{mmol} / \mathrm{mol}]$ vs. $11.0 \pm 2.5 \%$ [86 $\pm 4 \mathrm{mmol} / \mathrm{mol}], \mathrm{p}<0.001)$, total cholesterol $(227.2 \pm 47.3 \mathrm{mg} / \mathrm{dL}$ vs. $207.2 \pm 45.2 \mathrm{mg} / \mathrm{dL}$, $\mathrm{p}<0.001)$, LDL-C $(151.3 \pm 34.3 \mathrm{mg} / \mathrm{dL}$ vs. $139.8 \pm$ $\pm 27.8 \mathrm{mg} / \mathrm{dL}, \mathrm{p}<0.001)$, triglyceride $(199.5 \pm$ $\pm 118.4 \mathrm{mg} / \mathrm{dL}$ vs. $168.7 \pm 92.2 \mathrm{mg} / \mathrm{dL}, \mathrm{p}<0.001)$ levels were significantly decreased, after improving glycemic control. There was no significant change in HDL-C $(42.7 \pm 13.7 \mathrm{mg} / \mathrm{dL}$ vs. $42.2 \pm 14.8 \mathrm{mg} / \mathrm{dL}$, $\mathrm{p}=\mathrm{NS}$ ) levels.

A significant decrease in BNP (95.0 [4.0-1807] $\mathrm{ng} / \mathrm{L}$ vs. $52.0[2.1-987.0] \mathrm{ng} / \mathrm{L}, \mathrm{p}<0.001)$ levels was observed after improving glycemic control. Distribution of BNP levels before and after treatment is shown in Figure 1. The decrease in BNP levels was positively correlated with the decrease in $\mathrm{HbA1c}(\mathrm{r}=0.345, \mathrm{p}=0.003)$ and fasting plasma glucose $(r=0.366, p=0.002)$. There was no correlation between decrease in BNP levels and lipid parameters and blood pressure $(\mathrm{p}=\mathrm{NS})$.

\section{Discussion}

In this study, after glycemic control was achieved, significant decrease in BNP and lipid levels was observed. To the best of our knowledge, this is the first clinical study demonstrating the effect of glycemic control on BNP measurements.

In the previous studies, glycemic control affects lipids positively [3, 4]. Sánchez-Quesada et al. [1] reported that optimizing glycemic control in patients with type 2 diabetes promotes atheroprotective changes, including larger LDL size, decreased electronegative LDL, and a higher proportion of Lp-PLA2 activity in HDL. Wägner et

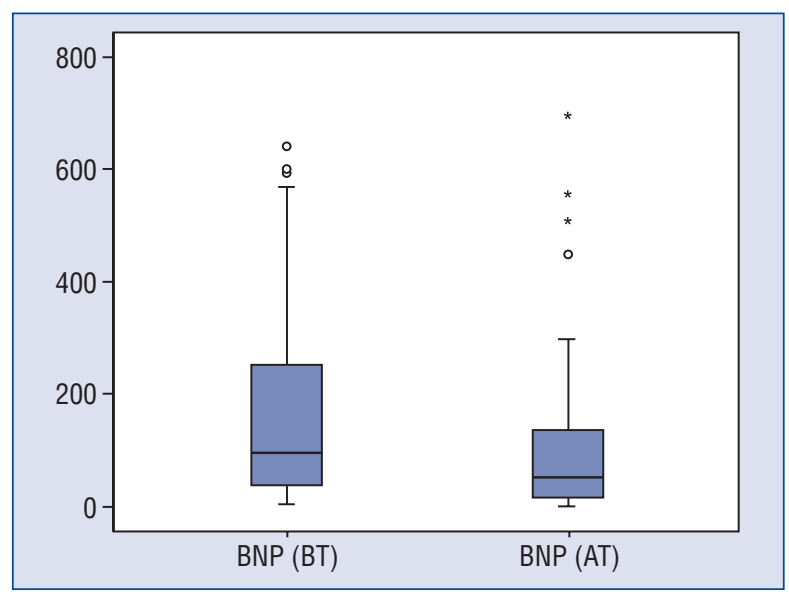

Figure 1. Distribution of B-type natriuretic peptide (BNP) levels before and after glycemic control; BNP (BT) BNP levels before treatment; BNP (AT) - BNP levels after treatment.

al. [2] demonstrated that improvement of glycemic control in type 2 diabetes improves most of the components of diabetic dyslipidemia, including a shift towards larger LDL particles in subjects with phenotype B.

Chronic heart failure (CHF) has been shown to be related to diabetes mellitus (DM). Diabetics have an increased risk of developing heart failure [7], and CHF is an independent risk factor for the development of DM [8, 9]. Cardiac functions are also associated with impairment of glucose metabolism [10]. The mechanisms underlying the relation between DM and CHF have not been fully elucidated. 
There are very few studies investigating the effect of hyperglycemia on BNP levels. In an experimental study, after 2 and 4 months of diabetes $\mathrm{db} / \mathrm{db}$ mice developed changes indicative of cardiac failure, and their myocardium showed increased mRNA expression of atrial and BNPs [11]. Treatment with macitentan (dual endothelin receptor antagonist) prevented from such abnormalities. Ortola et al. [12] found elevated atrial natriuretic peptide (ANP) concentrations in diabetic rats and they observed reduced glomerular filtration rate after infusion of ANP in diabetic rats. They concluded that elevated endogenous ANP levels contribute to the hyperfiltration observed in early diabetes in the rats [12].

Ciftel et al. [13], investigated the NT-proBNP levels in DM and control groups. They found significantly higher NT-proBNP levels in the diabetics with left ventricular diastolic dysfunction (LVDD) than the diabetics without LVDD, and normal controls. The mean NT-proBNP levels in the diabetics without LVDD were higher (albeit not significant) than in the control group. In another study with type $1 \mathrm{DM}$, glucose infusion caused elevation of ANP, but not BNP levels. They also showed in another study that plasma concentrations of ANP and BNP were elevated in patients with type $1 \mathrm{DM}$ $[14,15]$. Similarly to our study, they also found that plasma BNP concentrations were related to HbA1c. The same group has also demonstrated that BNP infusion can induce microalbuminuria in type $1 \mathrm{DM}$. In another study, BNP was found to be related to $\mathrm{HbA} 1 \mathrm{c}$ and QUICKI insulin sensitivity index in CHF patients [3].

The reason of the relation between BNP and hyperglycemia is not obvious. Hyperglycemia may be inducing dysfunction of the cardiac myocytes, and/or plasma glucose per se, may be inducing the secretion of cardiac natriuretic peptides. Endoplasmic reticulum stress associated with DM leads to cardiac myocyte apoptosis and cardiomyopathy as well $[16,17]$. Endoplasmic reticulum stress may be the link between elevated BNP secretion and DM.

\section{Conclusions}

We demonstrated a significant decrease in BNP and lipid levels by achieving glycemic control. This finding emphasizes the importance of glycemic control in diabetic patients. Poor glycemic control may cause high BNP measurements which can lead to overdiagnosis of CHF. We suggest that $\mathrm{HbA1c}$ and fasting plasma glucose should be checked in diabetic patients with high levels of BNP.

Conflict of interest: none declared

\section{References}

1. Sánchez-Quesada JL, Vinagre I, de Juan-Franco E et al. Effect of improving glycemic control in patients with type 2 diabetes mellitus on low-density lipoprotein size, electronegative low-density lipoprotein and lipoprotein-associated phospholipase A2 distribution._Am J Cardiol, 2012; 110: 67-71.

2. Wägner AM, Jorba O, Rigla M et al. Effect of improving glycemic control on low-density lipoprotein particle size in type 2 diabetes. Metabolism, 2003; 52: 1576-1578.

3. Vaverková H, Chlup R, Ficker L, Novotny D, Bartek J. Complementary insulin therapy improves blood glucose and serum lipid parameters in type 2 (non-insulin-dependent) diabetic patients. II. Effects on serum lipids, lipoproteins and apoproteins. Exp Clin Endocrinol Diabetes, 1997; 105 (suppl. 2): 74-77.

4. Taskinen MR, Kuusi T, Helve E, Nikkilä EA, Yki-Järvinen H. Insulin therapy induces antiatherogenic changes of serum lipoproteins in noninsulin-dependent diabetes. Arteriosclerosis, 1988; 8: 168-177.

5. Karadag O, Calguneri M, Yavuz B et al. B-type natriuretic peptide (BNP) levels in female systemic lupus erythematosus patients: what is the clinical significance? Clin Rheumatol, 2007; 26: 1701-1704 .

6. Stolz D, Breidthardt T, Christ-Crain M et al. Use of B-type natriuretic peptide in the risk stratification of acute exacerbations of COPD. Chest, 2008; 133: 1088-1094.

7. Nichols GA, Gullion CM, Koro CE, Ephross SA, Brown JB. The incidence of congestive heart failure in type 2 diabetes: An update. Diabetes Care, 2004; 27:1879-1884.

8. Egstrup M, Schou M, Gustafsson I, Kistorp CN, Hildebrandt PR, Tuxen CD. Oral glucose tolerance testing in an outpatient heart failure clinic reveals a high proportion of undiagnosed diabetic patients with an adverse prognosis. Eur J Heart Fail, 2011; 13: 319-326.

9. Clodi M, Resl M, Stelzeneder D et al. Interactions of glucose metabolism and chronic heart failure. Exp Clin Endocrinol Diabetes, 2009; 117: 99-106.

10. Shimabukuro M, Higa N, Asahi $\mathrm{T}$ et al. Impaired glucose tolerance, but not impaired fasting glucose, underlies left ventricular diastolic dysfunction. Diabetes Care, 2011; 34: 686-690.

11. Sen S, Chen S, Feng B, Iglarz M, Chakrabarti S. Renal, retinal and cardiac changes in type 2 diabetes are attenuated by macitentan, a dual endothelin receptor antagonist. Life Sci, 2012; 91: 658-668.

12. Ortola FV, Ballermann BJ, Anderson S, Mendez RE, Brenner BM. Elevated plasma atrial natriuretic peptide levels in diabetic rats. Potential mediator of hyperfiltration. J Clin Invest, 1987; 80: 670-674.

13. Ciftel S, Icagasioglu S, Yildiz G, Tekin G, Aydin H. Association of left ventricular diastolic dysfunction with elevated NT-proBNP in type 2 diabetes mellitus patients with preserved ejection fraction: The supplemantary role of tissue doppler imaging parameters and NT-proBNP levels. Diabetes Res Clin Pract, 2012; 96: 179-186.

14. McKenna K, Smith D, Tormey W, Thompson CJ. Acute hyperglycaemia causes elevation in plasma atrial natriuretic peptide concentrations in type 1 diabetes mellitus. Diabet Med, 2000; 17: 512-517.

15. McKenna K, Smith D, Sherlock M et al. Elevated plasma concentrations of atrial and brain natriuretic peptide in type 1 diabetic subjects. Ir J Med Sci, 2005; 174: 53-57.

16. Lakshmanan AP, Meilei H, Suzuki K et al. The hyperglycemia stimulated myocardial endoplasmic reticulum (ER) stress contributes to diabetic cardiomyopathy in the transgenic non-obese type 2 diabetic rats: A differential role of Unfolded Protein Response (UPR) signaling proteins. Int J Biochem Cell Biol, 2013; 45: 438-447.

17. Xu J, Zhou Q, Xu W, Cai L. Endoplasmic reticulum stress and diabetic cardiomyopathy. Exp Diabetes Res, 2012; 2012: 827-971. 